\title{
DERECHO Y POLÍTICAS AMBIENTALES EN ASTURIAS (SEGUNDO SEMESTRE 2021)
}

\author{
ALEJANDRA BOTO ÁLVAREZ \\ Profesora Titular de Derecho Administrativo. \\ Universidad de Oviedo
}

\begin{abstract}
Sumario: 1. Textos legales en tramitación. 1.1. Una Ley ambiental en ciernes. 1.2. Actividad física y deportiva en perspectiva ambiental. 2. Decretos aprobados. 2.1. Consejo de la Movilidad. 2.2. Instituto Mixto de Investigación en Biodiversidad (IMIB). 2.3 Registro de huella de carbono para la reducción, absorción y compensación de emisiones de gases de efecto invernadero. 2.4 Desempeño de las competencias en materia de saneamiento de agua. 2.5. Consejo para la Agenda 2030. 3. Decretos en preparación. 3.1. Caza. 3.2. Turismo. 4. Planes y programas. 4.1. Medidas concretas para la protección de la fauna. 4.2 Lobo ibérico: ¿statu quo? 4.3. Plan Director de Abastecimiento de Aguas 2020-2030. 4.4. Plan de adaptación al cambio climático de los puertos autonómicos.
\end{abstract}

El período que cubre la presente crónica ha sido especialmente activo desde la perspectiva de la normativa con incidencia ambiental, habiéndose iniciado la tramitación de una esperada Ley autonómica general sobre medio ambiente, y de otros instrumentos sectoriales de rango reglamentario. También se ha dado continuidad a líneas de trabajo que estaban abiertas y de las que crónicas precedentes habían dado oportuna noticia, a la que conviene dar seguimiento. A fin de realizar una presentación sistemática de estas cuestiones, se opta por su presentación agrupada en atención al rango del instrumento que les afecta, y a su estado de avance.

\section{TEXTOS LEGALES EN TRAMITACIÓN}

\subsection{Una Ley ambiental en ciernes}

En el BOPA núm. 162, de 23 de agosto de 2021 se publicaba el arranque del trámite de información pública respecto al Anteproyecto de Ley del Principado de Asturias de "calidad ambiental". Tal es finalmente la expresión elegida como título para una norma que buscar servir de marco general para la protección y 
tutela del medio ambiente en la Comunidad Autónoma ${ }^{1}$, mediante el establecimiento de los fines y principios a que debe quedar sujeta la actividad económica, tanto pública como privada, por razones medioambientales, de forma que se contribuya a lograr un crecimiento sostenible.

La consulta pública previa sobre la iniciativa, lanzada en julio de 2020, identificaba su necesidad de aprobación sin alternativas, pues hasta ahora, para el desarrollo de proyectos y actividades con incidencia sobre el medio, se viene aplicando en Asturias la legislación estatal (por ejemplo, en materia de evaluación ambiental y de autorizaciones ambientales), pero estos procedimientos resultan en ocasiones demasiado abiertos y generales. Por razones de eficacia administrativa, y coordinación, es necesario su desarrollo normativo. Este, requiere de una norma con rango de Ley pues, por ejemplo, es preciso solucionar la necesaria derogación de la figura de la Evaluación Preliminar de Impacto Ambiental (EPIA), establecida en la Ley 5/1991, de 5 de abril, de Protección de los Espacios Naturales del Principado de Asturias, que no tiene encaje en el marco normativo europeo actualmente vigente en este ámbito. También es necesario aclarar el régimen de las actividades clasificadas, que siguen rigiéndose en esta Comunidad Autónoma aún por el Reglamento de Actividades Molestas, Insalubres, Nocivas y Peligrosas (RAMINP) del año 1961, lo que genera una importante litigiosidad que se ha analizado en varias ocasiones en la sección de análisis jurisprudencial de esta Revista. Otro objetivo declarado era dotar a la Comunidad Autónoma de una norma que posibilite la adopción de compromisos políticos en materia de planificación ambiental y el ulterior desarrollo reglamentario en aspectos concretos como el establecimiento de valores límite de emisión, la contaminación acústica, la normalización de la documentación de seguimiento a presentar por las empresas, o los instrumentos económicos al servicio de la protección ambiental. A pesar de la evidente

\footnotetext{
${ }^{1}$ Algunas noticias previas habían anunciado una "Ley de sostenibilidad y protección ambiental" y llegaron a circularse borradores con ese nombre: https://www.asturias.es/Asturias/SEDE/FICHEROS_SEDE/tablon/proyectos_normativos/13_16 1205_anteproyecto_ley_sostenibilidad.pdf.
} 
importancia estructural de la iniciativa, la consulta previa no suscitó ningún comentario ${ }^{2}$.

Prácticamente un año más tarde se ha hecho público oficialmente el texto del Anteproyecto $^{3}$, pendiente de recibir alegaciones en trámite de audiencia hasta el 16 de septiembre, y de información pública hasta el 22 del mismo mes. Se trata de un texto largo y prolijo, con 113 artículos distribuidos en seis títulos además del preliminar (información y participación pública; instrumentos para la mejora de la calidad ambiental; instrumentos de intervención administrativa; coordinación ente instrumentos; vigilancia, control e inspección; y disciplina ambiental), 7 disposiciones adicionales, 2 transitorias, 1 derogatoria y 9 finales.

El objetivo principal del texto propuesto es lograr un crecimiento sostenible, inteligente e inclusivo, posibilitando que se desarrollen actividades sin condicionar el futuro ambiental del territorio y sus futuras generaciones. Para ello la norma incorpora instrumentos voluntarios para la mejora de la calidad ambiental y lucha contra el cambio climático, como son el fomento de los sistemas de gestión medioambiental o el cálculo, reducción y compensación de la huella de carbono. Se impulsan asimismo las buenas prácticas en ecoinnovación y la economía circular, mediante herramientas como la compra verde y la fiscalidad ambiental, o el apoyo a la investigación y la educación ambiental. Se bebe de la regulación europea y estatal, pero se considera necesario una adaptación integrada al ámbito regional, que se refleje en un único cuerpo legal, en aras de una mayor racionalidad y claridad, garantizando así un mayor nivel de protección del derecho a la seguridad jurídica de todos aquellos interesados en promover una actividad, instalación, proyecto, plan o programa susceptible de afectar al medio ambiente.

La lógica que inspira el texto es la de regular nuevas formas de intervención administrativa que sean capaces de compatibilizar protección medioambiental con simplificación y agilización de los trámites administrativos. A esos efectos se

\footnotetext{
${ }^{2}$ Puede verse al respecto la información disponible a través del portal de participación pública: http://www.asturiasparticipa.es/consulta_previa/proyecto-de-ley-del-principado-de-asturias-decalidad-ambiental/\#comentarios.

3 También está disponible en el mismo portal: http://www.asturiasparticipa.es/informacion_publica/anteproyecto-de-ley-de-calidad-ambientaldel-principado-de-asturias/\#.
} 
propone desarrollar el sistema de intervención administrativa ambiental para las actividades e instalaciones de mayor incidencia, sometidas al régimen de la autorización ambiental integrada, por serles de aplicación la Ley de prevención y control integrados de la contaminación. Para aquellas otras actividades que tienen una moderada incidencia sobre el medio ambiente y que requieren una 0 varias autorizaciones ambientales sectoriales, ya sea porque tienen emisiones a la atmósfera, generan vertidos o porque tratan residuos, se establece el régimen de la autorización ambiental integrada simplificada, que tiene por objeto reunir en una única autorización autonómica las diferentes autorizaciones ambientales sectoriales, aplicando principios de simplificación, racionalización y cooperación entre Administraciones. Por último, para aquellas actividades e instalaciones con escasa incidencia ambiental, se introduce el régimen de declaración responsable ambiental, que posibilita a los titulares de las actividades iniciar su ejercicio sin necesidad de autorización o de otro acto administrativo previo, sustituyendo el control previo por un control posterior, con el efecto positivo de reducción de trámites y plazos para la implantación de la actividad. Se excluyen del ámbito de intervención administrativa ambiental, por su escasa incidencia ambiental, las actividades sujetas a la Ley 8/2002, de 21 de octubre, de espectáculos públicos y actividades recreativas del Principado de Asturias, así como las actividades comerciales minoristas y la prestación de servicios incluidos en el ámbito de aplicación de la Ley 12/2012, de 26 de diciembre, de medidas urgentes de liberalización del comercio y de determinados servicios.

Seguiremos informando sobre la suerte de este texto, que está llamado a tener un protagonismo evidente en la protección ambiental, aunque no es el único con incidencia en la materia que está en tramitación; ocurriendo que, paradójicamente, son otros los que hasta ahora han sabido movilizar en mayor medida a la sociedad asturiana.

\subsection{Actividad física y deportiva en perspectiva ambiental}

En el BOPA núm. 83, de 3 de mayo de 2021 se lanzó por su parte el trámite de información pública sobre un texto referido a un Anteproyecto de Ley del Principado de Asturias de actividad física y deporte, iniciativa en la que ya se venía trabajado desde la Comunidad Autónoma desde 2018. La consulta pública previa abierta en aquel primer momento había puesto de manifiesto no pocas 
inquietudes de actores sociales en relación con la práctica deportiva en la naturaleza, en particular respecto a la eventual consideración como instalaciones deportivas en la naturaleza de senderos y vías de escalada ${ }^{4}$. Vuelto a abrir el proceso en el año 2020, el impacto ambiental del deporte en la naturaleza volvió a suscitar comentarios desde la perspectiva del montañismo y de los rescates (y su coste) ante accidentes deportivos en el medio natural ${ }^{5}$.

El texto dado a conocer pretende llevar la actividad física y deportiva al conjunto de la sociedad asturiana, adoptando una política deportiva global, que se esfuerce en propagar los valores sociales, educativos, formativos, de salud, cívicos, solidarios, de cohesión e integración, de desarrollo económico, turístico y medioambiental a todas las capas de la población. Uno de sus principios rectores es la promoción de la actividad física y deportiva en el medio natural como espacio deportivo, haciendo la práctica deportiva compatible y sostenible con la protección del medio ambiente. También busca velar por la existencia de información actualizada de la regulación y de las condiciones y lugares donde se puede realizar la práctica deportiva en el medio natural, e implementar políticas públicas transversales con otros sectores afectados, tales como la salud, la educación, la igualdad de oportunidades entre mujeres y hombres, la ordenación del territorio, el medio ambiente o el turismo, dado el interés público inherente a la actividad física y el deporte, en orden a conseguir una gestión de la actividad física y el deporte eficaz y eficiente, sostenible y transparente.

La necesidad de tomar conciencia de la huella ambiental de actividades como el deporte y el turismo están marcando la actualidad de los trabajos normativos también de rango jerárquico inferior en Asturias, como se verá en los apartados siguientes.

\section{DECRETOS APROBADOS}

\subsection{Consejo de la Movilidad}

\footnotetext{
${ }^{4}$ Pueden verse las observaciones de la Federación de Deportes de Montaña, Escalada y Senderismo del Principado de Asturias en http://www.asturiasparticipa.es/consulta_previa/anteproyecto-de-ley-de-actividad-fisica-ydeporte/\#.

5 http://www.asturiasparticipa.es/consulta_previa/anteproyecto-de-ley-de-actividad-fisica-ydeporte-2/.
} 
La Ley del Principado de Asturias 12/2018, de 21 de noviembre, de transportes y movilidad sostenible, que en su momento ya fue comentada en esta Revista ${ }^{6}$, establece en su artículo 25 que el Consejo de la Movilidad del Principado de Asturias es el órgano superior de participación, asesoramiento, consulta y debate sectorial en los asuntos que afecten al funcionamiento del sistema de transportes. Se dispone también que los órganos del Consejo, su composición y reglas de funcionamiento se determinarán reglamentariamente.

La disposición adicional sexta de la citada norma establece que el Consejo de Transportes Terrestres del Principado de Asturias regulado por el Decreto 112/2006, de 5 de octubre, cambiará su denominación por la de Consejo de la Movilidad del Principado de Asturias. Con todo, finalmente se ha decidido elaborar una nueva norma reguladora, por la necesidad de modificar las secciones del Consejo de Transportes Terrestres, con la inclusión de otros modos de transportes no recogidos en la norma anterior, así como la de los representantes de estos modos y de la Junta General del Principado de Asturias.

La nueva regulación se contiene en el Decreto 31/2021, de 23 de junio, publicado en el BOPA núm. 130, de 7 de julio. En ese mismo boletín se publicaría también otro Decreto con cierta relevancia medio ambiental, que se comenta a continuación.

\subsection{Instituto Mixto de Investigación en Biodiversidad (IMIB)}

EI Decreto 32/2021, de 23 de junio, crea el IMIB y supone la continuación a diez años de colaboración entre la Administración del Principado de Asturias, el Consejo Superior de Investigaciones Científicas (CSIC) y la Universidad de Oviedo, desde que el 18 de enero de 2011 firmaran un convenio de colaboración para la creación temporal de una Unidad Mixta de Investigación en Recursos Naturales y Biodiversidad de la Cordillera Cantábrica (UMIB).

Tras toda esa experiencia, las partes comparten la idea de que debe continuarse con esta iniciativa científica y dotarla de una mayor estabilidad e institucionalidad, para lo que se constituye este Instituto, que se crea con la finalidad de llevar a cabo, en un marco de colaboración, actividades de

\footnotetext{
${ }^{6}$ Vol. X, núm. 1 (2019).
} 
investigación, formación, transferencia y divulgación en el ámbito de la biodiversidad y cambio global, promoviendo un enfoque interdisciplinar.

\subsection{Registro de huella de carbono para la reducción, absorción y compensación de emisiones de gases de efecto invernadero (GEI)}

A través del Decreto 40/2021, de 29 de julio (BOPA núm. 149, de 3 de agosto), se regula la organización y funcionamiento de un registro autonómico que fomente y visibilice las iniciativas de medición y reducción de la huella de carbono de organismos, entidades y empresas, así como su compensación mediante créditos de carbono generados, principalmente, en proyectos de absorción de emisiones GEI desarrollados en la propia Comunidad Autónoma y que formarán parte del fondo de carbono regional.

La inscripción en el registro está dirigida a organizaciones públicas o privadas, así como a personas físicas, que deseen participar. En este sentido, atendiendo a su importancia en el tejido productivo asturiano, se dará especial relevancia a las contribuciones de pequeñas y medianas empresas, así como a organismos, Administraciones públicas y entidades del tercer sector, cuyo papel ejemplificante es de gran valor en la respuesta ante la emergencia climática.

El Decreto también establece los mecanismos necesarios para garantizar la compatibilidad del registro autonómico con el registro estatal regulado en el Real Decreto 163/2014, de 14 de marzo, de cara a la transmisión, trazabilidad y gestión de datos.

\subsection{Desempeño de las competencias en materia de saneamiento de agua}

Para el desempeño de las competencias en materia de saneamiento de agua, la Ley $1 / 1994$, de 21 de febrero, sobre abastecimiento y saneamiento de aguas en el Principado de Asturias, creó en su artículo 22 la Junta de Saneamiento como organismo autónomo adscrito a la Consejería competente en esa materia. El organismo, con personalidad jurídica propia e independiente, plena capacidad de obrar, patrimonio propio y autonomía funcional para el cumplimiento de sus funciones, vio su régimen jurídico positivizado en el Decreto 19/1998, de 23 de abril, por el que se aprueba el Reglamento para el desarrollo de la Ley 1/1994 del Principado de Asturias. 
Más de 25 años después, un objetivo racionalización administrativa, en aras de una mejora en la eficiencia de la gestión y en la coordinación y unidad de acción en materia de planificación, ejecución y explotación de las infraestructuras de saneamiento y depuración de aguas residuales, llevó a considerar oportuno la supresión del organismo autónomo Junta de Saneamiento, y la asunción de sus funciones por parte de los órganos centrales de la Administración del Principado de Asturias. La decisión se acometió a través de la Ley del Principado de Asturias 3/2020, de 30 de diciembre, de Presupuestos Generales para 2021, que en su disposición adicional duodécima suprime la Junta de Saneamiento, con efectos anteriores, en todo caso, al 31 de diciembre de 2021. Además, señala el párrafo 2, que las funciones desarrolladas por la Junta de Saneamiento pasarán a ser desempeñadas por la Administración del Principado de Asturias, a través de la Consejería de Administración Autonómica, Medio Ambiente y Cambio Climático.

En cumplimiento de esa previsión, en el BOPA núm. 155, de 11 de agosto de 2021 se publicó el Acuerdo del Consejo de Gobierno de 4 de agosto por el que se declara extinguida la personalidad jurídica del organismo autónomo Junta de Saneamiento, y también el Decreto 42/2021, de 4 de agosto, de segunda modificación del Decreto 33/2020, de 2 de julio, por el que se establece la estructura orgánica básica de la Consejería de Administración Autonómica, Medio Ambiente y Cambio Climático, que refleja el consiguiente cambio organizativo.

\subsection{Consejo para la Agenda 2030}

El último de los Decretos aprobados que se comentarán en este apartado tiene también un cariz organizativo. Responde a la decisión del Principado de Asturias de dotarse de una estructura ad hoc que, con vocación instrumental y marcado carácter técnico, coordine la ejecución de las actividades e iniciativas que se adopten en las materias relativas a la Agenda 2030, alineando los proyectos elegibles en su marco con las áreas y políticas estratégicas previamente definidas. Para ello, se considera necesario un órgano colegiado de consulta y participación, con el objetivo de prestar asesoramiento y colaboración institucional de la Administración del Principado de Asturias y la sociedad civil para el cumplimiento de los ODS y la Agenda 2030. 
Este órgano se crea mediante el Decreto 53/2021, de 20 de agosto, por el que se regula la organización y el funcionamiento del Consejo para la Agenda 2030 del Principado de Asturias. Entre sus funciones está asesorar a la Consejería competente en la elaboración e implementación de los planes y estrategias necesarios para el cumplimiento de la Agenda 2030; generar documentos y análisis sobre aspectos de su implementación; contribuir a la divulgación y comunicación de estos asuntos al conjunto de la ciudadanía asturiana, e impulsar el diálogo y coordinación entre todos los agentes sociales, económicos, medioambientales y culturales para contribuir a la consecución de los ODS.

Son miembros del Consejo, como presidente, la persona titular de la Consejería; como vicepresidente, la persona titular de la Dirección General competente en materia de Agenda 2030, y 23 vocales. Estos incluyen 6 representantes del sector empresarial y sindical 7 , 2 representantes del sistema universitario y centros de investigación ${ }^{8}, 6$ representantes de las Entidades del Tercer Sector, previa convocatoria de la Consejería, 6 representantes de Asociaciones y Fundaciones, previa convocatoria de la Consejería, 2 expertos independientes en el ámbito del desarrollo sostenible y la Agenda 2030, a propuesta de la persona titular de la Dirección General competente en materia de Agenda 2030 y la persona titular de la Presidencia de la Federación Asturiana de Concejos (FACC).

\section{DECRETOS EN PREPARACIÓN}

\subsection{Caza}

En el BOPA núm. 126, de 1 de julio, se publicaba anuncio abriendo a información pública una propuesta de Decreto de sexta modificación del Reglamento de caza

\footnotetext{
${ }^{7}$ La persona titular de la presidencia de la Federación Asturiana de Empresarios; la persona titular de la Secretaría General de la Unión General de Trabajadores en Asturias; la persona titular de la Secretaría General de Comisiones Obreras en Asturias; una persona en representación y a propuesta de las Cámaras de Comercio de Asturias; una persona en representación de otras organizaciones de carácter empresarial o económico y otra persona en representación de otras organizaciones de carácter sindical, previa convocatoria de la Consejería. Estas organizaciones, de carácter empresarial o económico y de carácter sindical, deberán tener su domicilio social o delegación autónoma en el territorio del Principado de Asturias, así como contemplar en sus estatutos como fines, alguno de los ámbitos relacionados con los ODS contenidos en la Agenda 2030.

${ }^{8}$ La persona titular del Rectorado de la Universidad de Oviedo; y una persona en representación de las organizaciones que trabajan en el ámbito de la investigación, a propuesta del Consejo Asturiano de Ciencia, Tecnología e Innovación.
} 
aprobado por Decreto 24/1991, de 7 de febrero. La iniciativa, sometida a consulta previa unos meses antes, había suscitado bastante participación social, y buscaba adecuar la ordenación de los aprovechamientos cinegéticos a la realidad socioeconómica actual de los concejos asturianos' ${ }^{9}$. El objetivo del texto es adaptar el Decreto a la situación demográfica de la región, caracterizada por una pérdida de población en el ámbito rural, un envejecimiento paulatino de la población residente y una pérdida de vinculación con la actividad cinegética que dificulta el relevo generacional. Por ello se modifica el requisito mínimo sobre el número de socios para acceder a los concursos tendentes a adjudicar el aprovechamiento de los cotos regionales. Igualmente se pretende adaptar el formato económico del Reglamento, modificando aquellos artículos en los que todavía se mantiene la mención a las pesetas y actualizando las cantidades para el pago del canon cinegético a los Ayuntamientos donde se ubican las Reservas Regionales de Caza. En aras de la seguridad jurídica es necesario que el desarrollo de la actividad de caza y sobre todo las limitaciones a su ejercicio tengan el mayor grado de concreción posible. A estos efectos, se publicarán por la Consejería con competencia en la materia los horarios hábiles establecidos para cada mes del año para el ejercicio de la caza y se incluye un nuevo artículo sobre las medidas de seguridad durante el desarrollo de las cacerías. Igualmente se intentan equiparar, siempre en la medida de lo posible, las cacerías en las Reservas a las de los Cotos, en cuanto al número de participantes, tanto de cazadores como de monteros y perros, y en este sentido, se incluyen cambios sobre el número máximo de cazadores en modalidad de batida (hasta veinte), en el número máximo de batidores (hasta diez) y en el número máximo de perros (hasta diez). Por último, debido a los avances tecnológicos y a la experiencia práctica, es conveniente modificar la obligación de supervisión y acompañamiento de los guardas durante toda la cacería, suprimiendo esta carga que a día de hoy es innecesaria. Por este motivo, en la modalidad de batida, se considerará suficiente la presencia del guarda al inicio y al final de la misma, con el objeto de realizar las verificaciones pertinentes.

\footnotetext{
9 http://www.asturiasparticipa.es/consulta_previa/proyecto-de-decreto-de-sexta-modificaciondel-reglamento-de-caza-aprobado-por-decreto-24-1991-de-7-de-febrero/. El vigente Plan de caza de las Reservas regionales de caza para la temporada 2021-2022 se publicó en el BOPA núm. 74, de 20 de abril de 2021.
} 


\subsection{Turismo}

En los últimos meses se han lanzado distintas iniciativas de reformas reglamentarias sectoriales en turismo que tienen un marcado impacto ambiental, por lo que habrá que seguir de cerca su devenir.

Se trata, en particular y entre otras, de sendas iniciativas en materia de albergues; campamentos y caravanas; y turismo activo. En los dos primeros casos ya existe un texto difundido en trámite de audiencia e información pública, mientras que en cuestión de turismo activo sólo se ha iniciado el trámite de consulta previa ${ }^{10}$. En los proyectos se deja claro que los albergues turísticos deberán cumplir las disposiciones vigentes en materia de urbanismo, construcción y edificación, ordenación del territorio, medio ambiente, biodiversidad, consumo, salud pública y seguridad alimentaria, prevención contra incendios, accesibilidad, y cualesquiera otras que sean de aplicación ${ }^{11}$. Por su parte, y respecto a la acampada o turismo itinerante, existen importantes retos en materia de sostenibilidad, protección y salvaguarda de los recursos naturales y medioambientales existentes ${ }^{12}$. Se está trabajando en ellos en el marco de una amplia Estrategia de Turismo del Principado de Asturias en el horizonte 2020-2030, que se centra en alcanzar la calidad y excelencia de la Comunidad Autónoma como destino turístico de referencia, desarrollando el

\footnotetext{
10 http://www.asturiasparticipa.es/consulta_previa/proyecto-de-decreto-de-modificacion-parcialdel-decreto-111-2014-de-26-de-noviembre-de-turismo-activo-bopa-4-de-diciembre-de-2014/.

11 http://www.asturiasparticipa.es/informacion_publica/proyecto-de-decreto-de-ordenacion-delos-albergues-turisticos/. Junto a los aspectos ambientales, la cuestión de la accesibilidad tiene su relevancia, dado que la falta de regulación actual en la Comunidad Autónoma ha llevado a que por ejemplo se pretenda combatir ante los tribunales la denegación de una licencia por razones vinculadas a las barreras arquitectónicas, bajo el argumento de que "el albergue está orientado a los peregrinos que recorren el Camino (...) de por sí inaccesible (...) para aquellos peregrinos con algún tipo de discapacidad". EI TSJA no acoge esta defensa, sosteniendo que la normativa legal aplicable ha de ser observada en la obra en virtud de la cual se solicita la licencia, sin que pueda escudarse para su incumplimiento en otros edificios o bien otras partes ajenas a la obra y proyecto pretendido (sentencia 551/2021, de 31 de mayo, en recurso de apelación 20/2021). La relación de ciertos aspectos ambientales con cuestiones turísticas y urbanísticas es evidente, y en ese sentido merece la pena reseñar que el Principado de Asturias ha arrancado también los trabajos para elaborar una nueva normativa de ordenación integral del territorio. Entre sus objetivos está la creación de herramientas jurídicas que permitan preservar y acondicionar el territorio y los entornos construidos, para que se comporte de manera más resiliente ante los cambios ambientales que conocemos, o están por llegar, mediante la aplicación de datos científicos sobre las consecuencias del cambio climático y tomar medidas que nos permitan mitigarlo y adaptarnos. Permaneceremos desde esta sección de la Revista también pendientes de la evolución de estos trabajos.

12 http://www.asturiasparticipa.es/informacion_publica/proyecto-de-decreto-de-ordenacion-decampamentos-de-turismo-y-areas-especiales-de-acogida-de-autocaravanas-en-transito/\#.
} 
sector desde una perspectiva sostenible, potenciando su ventaja competitiva, promoviendo una mayor generación y mejor distribución de riqueza, con especial protagonismo en la gobernanza, en el equilibrio territorial, social y económico así como en el mantenimiento y proyección de una excelente imagen de destino.

\section{PLANES Y PROGRAMAS}

\subsection{Medidas concretas para la protección de la fauna}

Se han aprobado en los últimos meses diferentes resoluciones en relación con ciertas especies que merecen ser destacadas, antes de atender de manera un poco más detallada al recurrente y polémico tema de la protección del lobo en un epígrafe separado.

Cabe citar aquí, por un lado, la aprobación el Programa de cría del poni de raza asturcón a través de la Resolución de 1 de junio de 2021, de la Consejería de Medio Rural y Cohesión Territorial, publicada en el BOPA núm. 122, de 25 de junio. El programa había sido presentado en diciembre de 2020 por la Asociación de criadores de poni de raza asturcón y se ajusta al Reglamento (UE) 2016/1012, del Parlamento europeo y del Consejo, de 8 de junio de 2016 y el Real Decreto $45 / 2019$, de 8 de febrero, por el que se establecen las normas zootécnicas aplicables a los animales reproductores de raza pura, porcinos reproductores híbridos y su material reproductivo y se actualiza el Programa nacional de conservación, mejora y fomento de razas ganaderas.

En cuanto a la fauna salvaje, en el BOPA núm. 92, de 14 de mayo, se publicó una resolución de 12 de abril de 2021, de la Consejería de Medio Rural y Cohesión Territorial, en orden de la protección del águila real, el alimoche común, el halcón peregrino y el buitre leonado durante sus períodos de reproducción y crianza, aumentando las zonas y fechas en que se prohíbe la escalada en ciertas áreas críticas para la especie.

Y finalmente, en el BOPA núm. 96, de 20 de mayo, se publicaba Resolución de 14 de mayo de 2021, de la Consejería de Medio Rural y Cohesión Territorial, por la que se establecen restricciones de acceso y tránsito para visitantes en áreas sensibles para el oso pardo en el Parque natural de las Ubiñas-La Mesa. 
Son ambas medidas que de nuevo evidencian las tensiones que las actividades recreativas generan en ocasiones en el medio natural, dependiendo del número de visitantes, la zona y la época del año.

\subsection{Lobo ibérico: ¿statu quo?}

En la entrega precedente de esta crónica se daba cuenta del cierto revuelo que en el Principado de Asturias habían causado diversos anuncios que apuntaban a la decisión ministerial de incluir el lobo ibérico en el Listado de Especies Silvestres en Régimen de Protección Especial. Mientras ese movimiento parecía haberse enfriado o al menos ralentizado, en la región se produjeron novedades, tanto desde el punto de vista político como jurisdiccional, que se comentan a continuación. Ninguna, conviene advertirlo ya, suponía un gran vuelco con respecto a la situación de la protección de la especie, pero la situación ha cambiado radicalmente a raíz de la aprobación de la Orden TED/980/2021, de 20 de septiembre, por la que se modifica el Anexo del Real Decreto 139/2011, de 4 de febrero, para el desarrollo del Listado de Especies Silvestres en Régimen de Protección Especial y del Catálogo Español de Especies Amenazadas (BOE núm. 226, de 21 de septiembre de 2021).

Por su parte, en el BOPA núm. 130, de 7 de agosto, había visto la luz la Resolución de 1 de julio de 2021, de la Consejería de Medio Rural y Cohesión Territorial, por la que se aprueba el Plan Anual de Actuaciones de Control del lobo 2021-2022. El estudio del plan da cuenta, como no podía ser de otra manera, de la creciente conflictividad social en torno a la especie. También se relatan experiencias piloto de cercados de protección. El plan autoriza en cualquier época y hasta el 31 de diciembre de 2022, la captura letal de 32 lobos en las siete áreas de gestión de las ocho en las que está zonificada la región. Fuera de dichas siete zonas, es decir, en el área de exclusión central y costero oriental, se podrán matar sin límite de ejemplares lobos previa autorización.

No habrá que esperar en exceso para saber si este plan, como otros anteriores, se acaba judicializando. Parece que así será a juzgar por algunos anuncios en redes sociales y prensa generalista de la Asociación para la Conservación y Estudio del Lobo Ibérico, que ha continuado muy activa en el planteamiento de distintas pretensiones ante los Tribunales. Las últimas tienen que ver con una 
serie de recursos contencioso-administrativos frente a distintos nombramientos en Direcciones Generales con competencia en la materia. La asociación ecologista combatía, con argumentos sustancialmente idénticos, los nombramientos por entender que se infringía lo dispuesto en el artículo 30 de la Ley del Principado de Asturias 8/2018, de 14 de septiembre, de Transparencia, Buen Gobierno y Grupos de Interés, debido a falta de idoneidad de las personas nombradas por ausencia de las condiciones legalmente establecidas al no gozar de formación y experiencia necesaria para el desempeño del cargo. A juicio de los recurrentes, ninguno de los Directores Generales integrados en la Consejería de Medio Rural y Cohesión Territorial tenía formación ni experiencia suficiente relacionados con la fauna animal, ni con el lobo ibérico, ni con su gestión pública, por lo que difícilmente implementarán de manera adecuada las medidas establecidas en el Plan del Lobo. Se argumentaba igualmente que con esos nombramientos se vulneraban los elementos reglados, que constituyen un límite de la discrecionalidad técnica en el nombramiento de altos cargos; y que faltaba motivación suficiente, incurriendo así en arbitrariedad, porque la motivación de los nombramientos no aparecía en el Decreto de nombramiento, sino en el informe de idoneidad del expediente, formulado además de manera muy genérica y como una fórmula-tipo que se repetía sin variación en todos los casos. Los recursos no tuvieron éxito, y en la medida en que la ratio decidendi no tuvo que ver con cuestiones ambientales sino de empleo público, no se ha considerado adecuado comentar estos fallos del TSJ de Asturias en la sección de jurisprudencia, bastando a mi juicio con que queden sucintamente expuestos aquí13.

Uno de los titulares de esas Direcciones Generales, en concreto la de ganadería, dimitiría 48 horas después de que el BOE publicara la inclusión del lobo ibérico en el Listado de Especies de Protección Especial ${ }^{14}$. El Principado ha anunciado que recurrirá la Orden Ministerial y que pedirá además medidas cautelares para

\footnotetext{
${ }^{13}$ El Tribunal acogió el argumento de la Administración, según el cual los recurrentes yerran al interpretar que deba darse un nexo indisoluble entre la formación y la experiencia de la persona nombrada con el campo de acción estricto de la materia sobre la que se vaya a ejercitar la Dirección General. Se trata de un conjunto de sentencias dictadas entre marzo y mayo de 2021. Por todas pueden verse las sentencias 483 y 484/2021, de 21 de mayo, que resuelven los recursos 950 y 952/2019, respectivamente.

${ }^{14}$ Su cese se dispuso por Decreto 63/2021, de 24 de septiembre, del Consejo de Gobierno (BOPA núm. 189, de 30 de septiembre).
} 
suspender su aplicación. En este punto debe tenerse en cuenta que la misma contiene dos disposiciones adicionales de gran interés transitorio. La primera señala que las medidas de extracción y captura de ejemplares que hayan adoptado los órganos competentes de las Comunidades Autónomas con anterioridad podrán seguir siendo de aplicación, siempre que se ajusten a las condiciones y a las limitaciones previstas en el artículo 61.1.b), c) y d) de la Ley 42/2007, de 13 de diciembre, del patrimonio natural y de la biodiversidad ${ }^{15}$, y en la Estrategia de conservación y gestión del lobo. La segunda, precisamente, emplaza a la Conferencia Sectorial de Medio Ambiente para la aprobación de esa Estrategia, antes del 31 de diciembre.

\subsection{Plan Director de Abastecimiento de Aguas 2020-2030}

El Gobierno asturiano prevé aprobar en octubre el Plan Director de Abastecimiento 2020-2030 que contará con una inversión de 423 millones. Está previsto que los proyectos que se incluyan sean financiados tanto a través del marco financiero plurianual de la Unión Europea 2021-2027 como del fondo Next Generation EU.

En el BOPA núm. 77, de 23 de abril, se publicó la apertura del plazo de información pública correspondiente a la evaluación ambiental estratégica del Plan. Su objetivo es garantizar la satisfacción de las demandas de agua

15 En concreto, se podrán aplicar medidas de extracción y captura de ejemplares que cuenten con una autorización administrativa que se conceda por la autoridad competente de la Comunidad Autónoma, siempre que cumplan los siguientes criterios: a) No exista otra solución satisfactoria, esto es cuando se haya demostrado que se han aplicado adecuadamente por parte de las explotaciones afectadas medidas preventivas o de protección del ganado, y estas hayan resultado ineficaces, teniendo para ello en cuenta el catálogo de medidas de protección del ganado ante eventos de depredación del lobo publicado por el Ministerio para la Transición Ecológica y el Reto Demográfico, así como otras medidas de protección que hayan sido previamente valoradas favorablemente por la comunidad autónoma o para las cuales se disponga de evidencia científica sobre su efectividad; b) Se justifique con el mejor conocimiento disponible que la medida de extracción y captura de ejemplares no afecta negativamente al estado de conservación favorable de la especie; y c) Se justifique la existencia de perjuicios importantes para el ganado en las explotaciones afectadas, atendiendo a posibles daños recurrentes o significativos. Las medidas deberán ser selectivas, realizarse en un tiempo lo más próximo posible al perjuicio y lo más cercano a las explotaciones afectadas y deberá realizarse un seguimiento de la efectividad de las actuaciones de extracción. Los órganos competentes de las Comunidades Autónomas comunicarán al Ministerio para la Transición Ecológica y el Reto Demográfico, a través de la Dirección General de Biodiversidad, Bosques y Desertificación, las autorizaciones administrativas concedidas, a los efectos y en los términos previstos en el apartado 6 del artículo 61 de la Ley 42/2007, de 13 de diciembre. 
abastecida de la población asturiana y de sus sectores económicos, tanto en cantidad como en calidad, así como contribuir a la protección de las masas de aguas naturales y del medio ambiente, mediante el respeto de los caudales ecológicos, el fomento del uso sostenible de los recursos hídricos y la recuperación de los costes de los servicios relacionados con el agua, incluidos los costes medioambientales. Será también un instrumento al que atender en crónicas futuras, al igual que el que se comenta a continuación, dando cierre ya a la presente.

\subsection{Plan de adaptación al cambio climático de los puertos autonómicos}

En el BOPA núm. 87, de 7 de mayo, se publicó por su parte el anuncio de la iniciación de un plan para adecuar las infraestructuras portuarias a los retos que plantea el calentamiento global del planeta y garantizar su operatividad en el futuro. El plan evaluará el nivel de riesgo al que están expuestos en la actualidad los puertos, y el incremento del mismo, producido por los impactos del clima, con dos horizontes temporales: 2050 y 2100 . Los factores a valorar serán, entre otros, el crecimiento de la inversión necesaria para la reparación de los daños causados en las infraestructuras, instalaciones y equipamiento por inundación costera, aumento del oleaje o la precipitación, la subida de los costes de mantenimiento, y la reducción de ingresos por parada operativa a causa del aumento de la precipitación, viento, temperatura, agitación, rebase o indisponibilidad del francobordo mínimo.

En función del nivel de riesgo climático estimado para cada puerto, el plan propondrá una serie de medidas para su reducción por debajo de los límites admisibles, con arreglo a un análisis coste-beneficio a corto, medio y largo plazo. Las medidas incluirán desde refuerzos de las estructuras de abrigo a la relocalización de determinadas actividades, pasando por dragados, reorganización o refuerzo de atraques, generación de energías renovables, implantación de sistemas de alerta temprana o mejora de la eficiencia energética de las instalaciones. 